\title{
Biochemical and Bioimpedance Correlation with Quality of Life and IIIness Perception in Hemodialysis Patients
}

\begin{abstract}
MIHAELA-DORA DONCIU1*, LAURA FLOREA ${ }^{1}$, LUANDA-IRINA MITITIUC ${ }^{1}$, ELENA-RODICA POPESCU², ROXANA CHIRITA ${ }^{2}$
${ }^{1}$ Grigore T. Popa University of medicine and Pharmacy, Faculty of Medicine, Department of Nephrology, 16 Universitatii Str., 700115, Iasi, Romania

${ }^{2}$ Grigore T. Popa University of Medicine and Pharmacy, Faculty of Medicine, Department of Psychiatry, 16 Universitatii Str., 700115, Iasi, Romania

In hemodialysis patients malnutrition-inflammation syndrome, evaluated by biochemical laboratory analysis and through bioimpedance spectroscopy, was associated to impaired quality of life and with an increased risk of mortality. The present study goal was to investigate, in a cross-sectional analysis, performed in 122 hemodialysis patients, the association between biochemical parameters and bioimpedance-derived measurements the association of undernutrition with quality of life and illness perception. The results showed that our cohort perceived an impaired quality of life and illness perception and that these psychometric evaluation was associated with malnutrition markers.
\end{abstract}

Keywords: quality of life, hemodialysis, bioimpedance spectroscopy

Chronic kidney disease (CKD) is a severe, global, health concern and it is associated with increased risk of all-cause mortality, cardiovascular (CV) mortality and impaired quality of life (QoL) [1]. In the same manner, end-stage renal disease (ESRD) and renal replacement therapy (RRT) are associated with decreased QoL, decreased daily physical activity and performance, severe metabolic and CV complication and a negative illness perception (IP) all of these disturbances leading to a significant social, familial and professional impact [2-6].

Furthermore, patients receiving chronic hemodialysis (HD) treatment, have an increased mortality risk due to protein-energy wasting (PEW) syndrome and chronic systemic inflammation fluid overload (FO) status [7-10]. Also, a negative IP and QoL were previously found to be correlated to with malnutrition-inflammation syndrome and chronic overhydration in HD population [9].

A recent method to correctly evaluate body composition in RRT patients, that is non-invasive, easy and fast, is represented by the bioimpedance spectroscopy (BIS) [11]. Using a Body Composition Monitor (BCM Fresenius), important, predictive nutritional parameters, as lean tissue mass (LTM), adipose tissue mass (ATM) and fat mass (FM) can be independently evaluated, regardless of the hydration status of the patient [11].

There are several reports that associate a low bodymass index (BMI), a low lean tissue index ( $\mathrm{LTI})$, overhydration expressed as measured overhydration $(\mathrm{OH})$, or as relative overhydration (Rel. $\mathrm{OH}$ ) as independent predictors for mortality in HD patients [9,12-15].

In the present study, we aimed to investigate the association of hematological, biochemical, clinical and BIS parameters with illness perception and quality of life in a cohort of hemodialysis patients.

\section{Experimental part}

\section{Matherial and Method}

Study Design and Patients Characteristics

An observational cross-sectional study was performed in stable HD patients in order to evaluate their illness perception and quality of life in association with hematological, biochemical, clinical, socio-demographical and BIS-derived parameters.
A total of 122 stable patients were included in the study ( 72 males and 50 females) who were receiving HD treatment three times weekly in Fresenius Nephrocare lasi Dialysis Unit. The exclusion criteria were: age under 18 years old, ongoing acute illnesses, psychiatric illnesses, patients with pace-makers, amputees and patients who declined to participate in the study. All patients gave informed consent prior to participation.

Socio-demographical, laboratory analysis, clinical, therapeutically and HD-related parameters

For all included participants, the following parameters were collected: age, gender, living environment, CKD etiology, comorbidities and chronic medication.

All blood test were collected in the last three weeks prior to the study's initiation and development; also, all laboratory analysis were collected before the start of the mid-week HD session. The analyzed parameters included: serum hemoglobin $(\mathrm{Hb})$ concentration, serum hematocrit (Ht) concentration, transferrin saturation (TSAT) values, serum alanine aminotransferase (ALT), serum alkaline phosphatase $(A P)$, serum potassium $(K)$, sodium $(\mathrm{Na})$, bicarbonate $\left(\mathrm{HCO}_{3}\right)$, phosphorus $(\mathrm{P})$, magnesium $(\mathrm{Mg})$, total calcium ( $\mathrm{CaT}$ ) concentrations, serum intact parathyroid hormone (PTH ) concentration, serum calciumphosphorus product (CaxP), serum $C$ reactive protein (CRP), serum ferritin, serum albumin, serum total cholesterol (CT), high-density lipoprotein cholesterol (HDLCT), low-density lipoprotein cholesterol (LDL-CT) and triglycerides (TG) concentrations.

Blood pressure (BP) measurements were performed the HD session, using, for the present study, an average measurement of $B P$ values obtained from the previous three consecutive HD sessions. HD-related parameters included: type of hemodialysis (conventional hemodialysis or hemodiafiltration (HDF)), dialysis vintage, vascular access (long-term catheter or arterio-venous fistula (AVF)) and dialysis adequacy expressed as $\mathrm{Kt} / \mathrm{V}$.

Assessment of body composition via BIS measurements

BIS measurements were obtained before the HD session, using a Body Composition Monitor (Fresenius Medical Care), a portable whole-body bioimpedance-

\footnotetext{
*email : mihaeladora1985@gmail.com; Phone: 0745918190
} 
analysis device working at 50 frequencies, over a range from 5 to $1000 \mathrm{KHz}$, in order to determine electrical tissue resistance and reactance (the high frequency current is able to pass through total body water (TBW), but the low frequency current can not pass through the cell membrane). The BCM measurements were obtained by trained medical staff, with the patient relaxed, in supine position, following an electrodes-placement protocol.

The following parameters were obtained: absolute, measured fluid overload $(\mathrm{OH})$, Rel. $\mathrm{OH}(\mathrm{OH} / \mathrm{ECW})$, extracellular water (ECW), intracellular water (ICW), ECW/ ICW ratio, total body water (TBW), lean tissue mass (LTM), fat mass (FM), adipose tissue mass (ATM), body cell mass $(\mathrm{BCM})$, body mass index $\mathrm{BMI})$, lean tissue index, defined as LTM/height ${ }^{2}$ (LTI), fat tissue index, defined as FTM/ height $^{2}$ (FTI), LTM/weight ratio (LTM/W), ATM to weight ratio (ATM/W).

For normal range intervals, previous age, gender and height values, from references population, both general population and HD patients, were established, with values defined for LTI and FTI as low if LTI, or FTI is $\leq 10$ th percentile of a reference population), high if LTI or FTI is ${ }^{3}$ $\geq 90$ th percentile and normal if LTI or FTI is between the 10th and the 90th percentile of the reference population [14]. Also, the references ranges for $\mathrm{OH}$ were established betw een $-1.1 \mathrm{I}$ and $1.1 \mathrm{l}$, values under this interval defining underhydration and overhydration, respectively. Rel. $\mathrm{OH}$ was also found to be statistically significant associated with mortality in renal population, a value $>15 \%$ being considered as severe overhydration being independently correlated with mortality in HD patients [15].

\section{Illness Perception Evaluation}

The Revised IIIness Perception Questionnaire (R-IPQ) was used in the study population. The R-IPQ comprises five scales to assess the cognitive representation of the illness: timeline of the disease, perceived as acute or chronic (TI), the cyclicity of the disease (CY), the perceived disease's consequences (CQ), the perceived personal control on the disease (PC), the perceived control that the received treatment for the underlying condition is exerting on the disease (TC), the personal coherence of the disease (CH) and a scale that assesses the emotional response (ER). A higher score on the TI scale indicates more chronic perceived course of the disease; a higher score on the $\mathrm{CY}$ scale indicates the patient's capacity to predict a given course of the disease; a higher score on the CQ scale indicates the patient's belief that the disease has severe life consequences; a higher score on the PC scale indicates the patient's belief that he/she has an efficient control on his/hers own disease; a higher score on the $\mathrm{CH}$ scale indicates that the patient is understanding the underlying disease and a higher score on the ER scale indicates a more significant, perceived emotional impact of the disease.

\section{Quality of Life Evaluation}

The Short Form Health Survey Questionnaire (SF-36) was used for assessing physical and mental measures in the study population. The SF-36 comprises eight dimensions, and two general domains, the Physical Component Summary (PCS) and the Mental Component Summary (MCS). The domains of SF-36 have a score ranging from 0 to 100, a higher score (towards 100) indicating a better functioning for that respective domain. The eightevaluated domains of the SF-36 are the following: Physical Functioning: the lowest score defines limitation in performing all physical activities, including daily, personal routine; Role-physical: the lowest score defines limitation in work or other activities as a result of physical impairment; Bodily Pain: the lowest score defines very severe and extremely limiting pain; General Health: the lowest score defines a personal health evaluation as being poor; Vitality: the lowest score defines tiredness all of the time; Social Functioning: the lowest score defines frequent, severe interference of the disease with normal social activities; Role-emotional: the lowest score defines problems with work and daily activities due to emotional disturbances; Mental Health: the lowest score defines constant feelings of nervousness and depression; Physical Component Summary: the lowest score defines limitations in self-care, social and role activities, severe bodily pain, frequent tiredness and a poor health-related evaluation; Mental Component Summary the lowest score defines frequent psychological distress, social and role disability due to emotional problems and a poor health-related evaluation.

Based on the two general scores, the PCS and the MCS, the included patients were further divided into three groups for each domain: the low-score PCS group (score between 0 and 49), the low-score MCS group (scores between 0 and 49), the medium-score PCS group (scores between 50 and 79), the medium-score MCS group (scores between 50 and 79 ) and the high-score PCS group (scores between 80 and 100) and the high-score MCS group (scores between 80 and 100), for the statistical analysis.

\section{Statistical Analysis}

All statistical analysis were performed using SPSS 20.0 statistical standard package for Mac OS X. Data were expressed as mean and standard deviation (SD) for continuous variables and as percentage with frequencies analysis for categorical variables. Bivariate correlation analysis, using Pearson's method was used for correlation between included variables. One -way analysis of variance ANOVA was used for categorical independent variables with more than two categories (the PCS type with three groups: low-scores, medium scores and high scores and the MCS type with three groups: low-scores, mediumscores and high-scores) and normally distributed interval dependent variables in order to test for differences in the means of the dependent variables, broken down by the levels of the independent variable. To evaluate the nature of the differences between the groups, a statistically significant ANOVA result was further followed by the Fisher's LSD post-hoc test. A statistically significant twotailed $p$ value of $<0.05$ was considered.

\section{Results and discussions}

There was a total number of 122 patients included in the study. Baseline characteristics of the study population are summarized in Tables 1, 2, 3, 4, 5 and 6 . Mean age was $56.84 \pm 13.92$ years, 72 patients (59\%) were males and 70 patients $(57.4 \%)$ lived in urban area. There were 47 patients (38.5\%) that received hemodiafiltration (HDF) while the remaining 75 patients $(61.5 \%)$ received conventional HD treatment; vascular access was represented, for 92 patients (75.4\%) by an arteriovenous fistula (AVF) and, for 29 patients (23.8\%) by a long-term catheter. The participants were receiving HD treatment for a mean of $72.97 \pm 64.87$ months, and the mean Kt/V was $1.33 \pm 0.30$, suggesting an adequate $H D$ treatment in the present cohort. Mean systolic blood pressure (SBP) was $148.89 \pm 19.62 \mathrm{mmHg}$ and mean diastolic blood pressure (DBP) was $77.01 \pm 11.98 \mathrm{mmHg}$ (table 1). The comorbidities in the study population and the chronic medication are described in tables 1 and 2. 
Table 1

SOCIO-DEMOGRAPHIC, CLINICAL, HD-RELATED PARAMETERS FOR THE STUDY POPULATION ( $\mathrm{N}=122$ )

\begin{tabular}{|c|c|}
\hline CHARACTERISTICS & MEAN $( \pm$ SD $)$ \\
\hline Age (years) & $56.84(13.92)$ \\
\hline Gender (male, \%) & $72(59 \%)$ \\
\hline Living Enviroment (urban \%) & $70(57.4 \%)$ \\
\hline \multicolumn{2}{|l|}{$\begin{array}{l}\text { Vascular Access: } \\
\text { nor }\end{array}$} \\
\hline Arteriovenous Fistula $(\mathbb{N}, \%)$ & $92(75.4 \%)$ \\
\hline Long-Term Catheter $(\mathbb{N} \%)$ & $29(23.8 \%)$ \\
\hline Hemodialysis Type (HDF, \%) & $47(38.5 \%)$ \\
\hline Dialysis Vintage (months) & $72.97(64.87)$ \\
\hline $\mathrm{Kt} / \mathrm{V}^{-}$ & $1.33(0.30)$ \\
\hline $\mathrm{SBP}(\mathrm{mmHg})$ & $148.89(19.62)$ \\
\hline $\mathrm{DBP}(\mathrm{mmHg})$ & $77.01(11.98)$ \\
\hline \multicolumn{2}{|l|}{ RENAL DISEASE ETIOLOGY } \\
\hline Chronic Glomerulonephritis $(\mathrm{N}, \%)$ & $41(33.6 \%)$ \\
\hline Nephroangiosclerosis $(\mathrm{N}, \%)$ & $9(7.4 \%)$ \\
\hline Ischaemic Nephropathy $(\mathrm{N}, \%)^{-}$ & $7(5.7 \%)^{-}$ \\
\hline Diabetic Nephropathy $(\mathbb{N}, \%)$ & $26(21.3 \%)$ \\
\hline $\mathrm{ADPKD}(\mathrm{N}, \%)^{-}$ & $13(10.7)$ \\
\hline Obstructive Nephropathy (N\%) & $5(4.1 \%)$ \\
\hline Tubulointerstitial Nephropathy $(\mathbb{N}, \%)$ & $16(13.1 \%)$ \\
\hline Lupus Nephropathy $(\mathbb{N}, \%)$ & $4(3.3 \%)$ \\
\hline \multicolumn{2}{|l|}{ COMORBIDITIES } \\
\hline Essential HTN $\left(\mathbb{N}^{\prime} \%\right)^{-}$ & $49(40.2 \%)$ \\
\hline Secondary HTN $(\mathbb{N}, \%)$ & $59(48.4 \%)$ \\
\hline \multicolumn{2}{|l|}{ Cardiovascular Disease: } \\
\hline Chronic Ischemic Heart Disease $(\mathbb{N}, \%)$ & $31(25.4 \%)$ \\
\hline Chronic Atrial Fibrillation $(\mathbb{N}, \%)$ & $16(13.1 \%)$ \\
\hline Dilated Cardiomyopathy $(N, \%)$ & $15(13.3 \%)$ \\
\hline Chronic Heart Failure $(\mathrm{N}, \%)$ & $17(13.9 \%)$ \\
\hline \multicolumn{2}{|l|}{ Diabetes Melitus: } \\
\hline Type $1 \mathrm{DM}(\mathrm{N} \% \%)$ & $5(4.1 \%)$ \\
\hline Type $2 \mathrm{DM}(\mathrm{N}, \%)$ & $26(21.3 \%)$ \\
\hline Virus C Hepatitis $(N, \%)$ & $26(21.3 \%)$ \\
\hline Secondary Anemia $(\mathrm{N}, \%)$ & $99(81.1 \%)$ \\
\hline Secondary Hyperparathyroidism $(N, \%)$ & $50(41 \%)^{--}$ \\
\hline
\end{tabular}

Mean Hb levels were $11.24 \pm 1.32 \mathrm{~g} / \mathrm{dL}$, mean C reactive protein (CRP) levels were $18.41 \pm 40.97 \mathrm{mg} / \mathrm{L}$, mean albumin levels were $4.69 \pm 0.61 \mathrm{~g} / \mathrm{dL}$, mean CT levels were $178.57 \pm 44.29 \mathrm{mg} / \mathrm{dL}$, mean HDL-CT levels were $42.06 \pm$ $16.38 \mathrm{mg} / \mathrm{dL}$, mean LDL-CT levels were $102.82 \pm 36.66$, mean TG levels were $174.19 \pm 110.79 \mathrm{mg} / \mathrm{dL}$ (table 3 ).

Table 4 summarizes BIS measurements. Mean LTM for the entire population was $35.47 \pm 9.53 \mathrm{~kg}$, mean FM was $27.57 \pm 13.64 \mathrm{~kg}$, mean ATM was $37.52 \pm 18.54 \mathrm{~kg}$, mean BMI was $27.18 \pm 5.89 \mathrm{~kg} / \mathrm{m}^{2}$, mean LTI was $12.86 \pm 2.77$ $\mathrm{kg} / \mathrm{m}^{2}$ and mean FTI was $13.76 \pm 6.72 \mathrm{~kg} / \mathrm{m}^{2}$.

The mean scores for R-IPQ and SF-36 evaluation are presented in Table 5 and 6 , respectively. In general, the included cohort showed a decreased quality of life, expressed by a mean PCS score of $45.12 \pm 23.69$ and a mean MCS score of $55.47 \pm 22.96$. Regarding illness perception examination, the lowest score was registered
Table 2

CHRONIC MEDICATION OF THE STUDY POPULATION ( $\mathrm{N}=122$ )

\begin{tabular}{|c|c|}
\hline CHARACTERISTICS & MEAN ( \pm SD) \\
\hline \multicolumn{2}{|l|}{ CHRONIC MEDICATION } \\
\hline Diuretics $(\mathrm{N}, \%)$ & $27(22.1 \%)$ \\
\hline $\mathrm{ACEI}_{5}(\mathrm{~N}, \%)$ & $10(8.2 \%)$ \\
\hline $\mathrm{ARB}_{5}(\mathrm{~N}, \%)$ & $2(1.6 \%)$ \\
\hline Beta-Blockers $(N, \%)$ & $52(42.6 \%)$ \\
\hline Calcium Channel Blockers $(\mathrm{N}, \%)$ & $32(26.2 \%)$ \\
\hline Nitrates $(\mathbb{N}, \%)$ & $10(8.2 \%)$ \\
\hline Antiarrhytmics $(\mathrm{N}, \%)$ & $8(6.6 \%)$ \\
\hline Antiaggregants $(\mathrm{N}, \%)$ & $57(46.7 \%)$ \\
\hline Statins $(N, \%)$ & $5(4.1 \%)$ \\
\hline $\mathrm{ESA}_{\mathrm{s}}(\mathrm{N}, \%)$ & $94(77 \%)$ \\
\hline IV Iron $(\mathrm{N}, \%)$ & $95(77.9 \%)$ \\
\hline \multicolumn{2}{|l|}{ Vitamine D Analogues: } \\
\hline Alpha $D_{3}(N, \%)$ & $73(59.8 \%)$ \\
\hline Paricalcitol $(\mathrm{N}, \%)$ & $16(13.1 \%)$ \\
\hline Calcimimetics $(\mathrm{N}, \%)$ & $7(5.7 \%)$ \\
\hline \multicolumn{2}{|l|}{ Phosphate Binders: } \\
\hline Sevelamer Carbonate $(\mathrm{N}, \%)$ & $8(6.6)$ \\
\hline Calcium acetate $\mathrm{Mg}$ carbonate $(\mathrm{N}, \%)$ & $21(17.2 \%)$ \\
\hline
\end{tabular}

Table 3

LABORATORY MEASUREMENTSFOR THE STUDY POPULATION ( $\mathrm{N}=122)$

\begin{tabular}{|c|c|}
\hline $\begin{array}{c}\text { HEMATOLOGICAL \& } \\
\text { BIOCHEMICAL PARAMETERS }\end{array}$ & MEAN ( \pm SD) \\
\hline Hemoglobin $(\mathrm{g} / \mathrm{dl})$ & $11.24(1.32)$ \\
\hline Hematocrit $(\%)$ & $34.32(4.09)$ \\
\hline TSAT (\%) & $28.57(14.41)$ \\
\hline ALT (IU/I) & $18(12.12)$ \\
\hline $\mathrm{AP}(\mathrm{IU} / 1)$ & $105.68(59.71)$ \\
\hline Potassium (Eq/1) & $5.05(0.78)$ \\
\hline Sodium $(\mathrm{mEq} / \mathrm{l})$ & $137.55(2.88)$ \\
\hline Phosphorus (mg/dl) & $4.54(1.30)$ \\
\hline $\mathrm{PTH}_{\mathrm{i}}(\mathrm{ng} / \mathrm{l})$ & $610.00(530.48)$ \\
\hline $\mathrm{CaxP}\left(\mathrm{mg} / \mathrm{dl}^{2}\right)$ & $39.68(11.84)$ \\
\hline Total Serum Calcium (mg/dl) & $8.73(0.73)$ \\
\hline Magnesium (mg/dl) & $2.50(0.34)$ \\
\hline $\mathrm{CRP}(\mathrm{mg} / \mathrm{l})$ & $18.41(40.97)$ \\
\hline Albumin, Alb (g/d) & $4.69(0.61)$ \\
\hline Ferritin (ng/1) & $781.15(554.13)$ \\
\hline $\mathrm{CT}^{-}(\mathrm{mg} / \mathrm{dl})$ & $178.57(44.29)$ \\
\hline HDL-CT (mg/d) & $42.06(16.38)$ \\
\hline $\mathrm{LDL}-\mathrm{CT}(\mathrm{mg} / \mathrm{dl})$ & $102.82(36.66)$ \\
\hline $\mathrm{TG}(\mathrm{mg} / \mathrm{dl})$ & $174.19(110.79)$ \\
\hline
\end{tabular}

by $\mathrm{CY}$ domain, with a mean value of $9.50 \pm 1.90$, suggesting that the included patients can not accurately predict the illness' course, or possible arising complications. Decreased score for illness perception were also observed for the TC and $\mathrm{CH}$ domains, with a mean value of $13.85 \pm$ 2.05 and $13.63 \pm 3.38$, respectively, suggesting that the included patients do not understand their disease and do not consider that HD treatment is efficiently controlling the ESRD pathology.

The bivariate correlation analysis showed thatPCS score was positively associated to the following parameters: $\mathrm{K}$ $(r=0.191 ; p<0.05), P^{2} H_{j}(r=0.187 ; p=0.05)$, albumin $(r$ $=0.191 ; p<0.05)$, HDL-CT $(r=0.217 ; p<0.05)$, ICW $(r=$ $0.183 ; p<0.05)$, TBW $(r=0.183 ; p<0.05), B C M(r=0.225$; $p<0.05)$, LTM $(r=0.236 ; p<0.05), \operatorname{LTI}(r=0.185 ; p<0.05)$, LTM/W $(r=0.255 ; p<0.05)$, TC $(r=0.283 ; p<0.05), C H(r$ $=0.54 ; p<0.01$ ) and was inversely associated to the following parameters: age $(r=-0.390 ; p<0.01)$, PLT $(r=$ $0.258 ; p<0.05)$, albumin $(r=-0.191 ; p<0.05), C T(r=-$ $0.200 ; p<0.05)$, TG $(r=-0.190 ; p<0.05)$, ECW/ICW $(r=-$ 
Table 4

BODY COMPOSITION MONITOR MEASUREMENTS FOR THE STUDY POPULATION ( $\mathrm{N}=122)$

\begin{tabular}{|c|c|}
\hline BIOIMPEDANCE PARAMETERS & MEAN $( \pm$ SD) \\
\hline Measured Overhydration, $\mathrm{OH}(\mathrm{l})$ & $1.30(1.47)$ \\
\hline Relative Overhydration, $\mathrm{OH} / \mathrm{ECV}$ & $7.37(8.25)$ \\
\hline Extracellular Water, ECW (1) & $18.50(19.53)$ \\
\hline Intracellular Water, ICW (I) & $18.26(4.62)$ \\
\hline ECW to ICW Ratio, ECW/ICW & $0.94(0.12)$ \\
\hline Total Body Water, TBW (I) & $34.95(7.22)$ \\
\hline Lean Tissue Mass, LTM (Kg) & $35.47(9.53)$ \\
\hline Adipose Tissue Mass, ATM(Kg) & $37.52(18.54)$ \\
\hline Fat Mass, FM (Kg) & $27.57(13.64)$ \\
\hline Body Cell Mass, BCM $(\mathrm{Kg})$ & $19.33(6.32)$ \\
\hline Body Mass Index, BMI $\left(\mathrm{Kg}^{2} \mathrm{~m}^{2}\right)$ & $27.18(5.89)$ \\
\hline Lean Tissue Index, LTI $\left(\mathrm{Kg} / \mathrm{m}^{2}\right)$ & $12.86(2.77)$ \\
\hline Fat Tissue Index, FTI $\left(\mathrm{Kg} / \mathrm{m}^{2}\right)$ & $13.76(6.72)$ \\
\hline LTM to Weight Ratio, LTM $/$ Wgt & $48.36(15.55)$ \\
\hline ATM to Weight Ratio, ATM/Vgt (\%) & $35.31(11.66)$ \\
\hline
\end{tabular}

Table 5

MEAN SCORES FOR THE R-IPQ FOR THE STUDY POPULATION ( $\mathrm{N}=122)$

\begin{tabular}{|c|c|}
\hline $\begin{array}{c}\text { REVISED ILLNES PERCEPTION } \\
\text { QUESTIONNAIRE (R-IPQ) DOMAINS }\end{array}$ & MEAN $( \pm$ SD) \\
\hline Timeline Acute/Chronic, II & $20.60(3.44)$ \\
\hline Timeline Cyclical, $\mathbf{C Y}$ & $9.50(1.90)$ \\
\hline Consequences, CQ & $16.43(2.70)$ \\
\hline Personal Control, PC & $14.10(1.92)$ \\
\hline Treatment Control, TC & $13.85(2.05)$ \\
\hline Illness Coherence, $\mathrm{CH}$ & $13.63(3.38)$ \\
\hline Emotional Response, ER & $14.78(4.64)^{-}$ \\
\hline
\end{tabular}

$0.220 ; p<0.05)$, BMI $(r=-0.181 ; p<0.05)$, FTI $(r=-0.180$; $p<0.05), A T M / W(r=-0.241 ; p<0.05), C Q(r=-0.664 ; p$ $=0.01), C Y(r=-0.363 ; p<0.01)$, ER $(r=-0.540 ; p<0.01)$.

The bivariate correlation analysis for MCS score showed a statistically significant positive correlation to the following parameters: PTH $(r=0.189 ; p<0.05), \mathrm{OH}(r=0.193$; $p<0.05), \mathrm{TC}(r=0.298 ; p<0.01), \mathrm{CH}(r=0.576 ; p<0.05)$ and a negative correlation to the following parameters: age $(r=-0.198 ; p<0.01), C T(r=-0.200 ; p<0.05)$, TG $(r=$ $-0.198 ; p<0.01)$, FTI $(r=-0.208 ; p<0.05) ; C Q(r=-0.687$; $p<0.01), C Y(r=-0.371 ; p<0.01)$; $E R(r=-0.650 ; p<0.01)$.

Correlations of socio-demographics, hematological, biochemical, BIS and R-IPQ parameters between the three $P C S$ groups (low-score, medium-score and high-score)

The independent between-groups ANOVA yielded a statistically significant effect for: age $(F(2.119)=8.53$; $p<0.01)$; HDL-CT $(F(2.119)=3.38 ; p<0.05), \operatorname{TI}(F(2.119)$ $=4.59 ; p<0.05), C Q(F(2.119)=35.84 ; p<0.01) ; T C$ $(F(2.119)=3.23 ; p<0.05), C H(F(2.119)=19.08 ; p<0.01)$; $C Y(F(2.119)=8.65 ; p<0.01) ; \operatorname{ER}(F(2.119)=19.31 ;$ $\mathrm{p}<0.01$ ) (table 7).

The post-hoc test showed the following: the differences between the low-score PCS group and the high-score PCS group were statistically significant for: age $(t(119)=13.35$; $\mathrm{p}=0.00), \mathrm{HDL}-\mathrm{CT}(\mathrm{t}(119)=-11.13 ; \mathrm{p}=0.01), \mathrm{TI}(\mathrm{t}(119)$ $=2.34 ; \mathrm{p}=0.01), \mathrm{CQ}(\mathrm{t}(119)=4.68 ; \mathrm{p}=0.00), \mathrm{TC}(\mathrm{t}(119)$ $=-1.25 ; \mathrm{p}=0.02), \mathrm{CH}(\mathrm{t}(119)=-4.22 ; \mathrm{p}=0.00), \mathrm{CY}(\mathrm{t}(119)$ $=2.06 ; p=0.00)$, ER $(t(119)=5.29 ; p=0.00)$. The differences between low-score PCS group and the medium-score PCS group were statistically significant for: age $(t(119)=7.41 ; p=0.00), C Q(t(119)=2.21 ; p=$ $0.00), \mathrm{CH}(\mathrm{t}(119)=-2.81 ; \mathrm{p}=0.00), \mathrm{ER}(\mathrm{t}(119)=4.32 ; \mathrm{p}$ $=0.00)$. The differences between the medium-score PCS group and the high-score PCS group were statistically
Table 6

SF-36 MEAN SCORESFOR THE STUDY POPULATION $(\mathrm{N}=122)$

\begin{tabular}{|c|c|}
\hline $\begin{array}{l}\text { QUALITY OF LIFE (SF-36) } \\
\text { DOMAINS }\end{array}$ & $\operatorname{MEAN}( \pm$ SD) \\
\hline Physical Functioning & $48.32(28.67)$ \\
\hline Role-Physical & $39.75(41.50)$ \\
\hline Bodily Pain & $50.27(31.34)$ \\
\hline General Health & $38.70(24.10)$ \\
\hline Vitality & $48.85(23.25)$ \\
\hline Social Functioning & $63.90(31.48)$ \\
\hline Role-Emotional & $59.03(41.32)$ \\
\hline Mental Health & $66.25(21.63)$ \\
\hline Physical Component Score, PCS & $45.12(23.69)$ \\
\hline Mental Component Score, MCS & $55.47(22.96)$ \\
\hline
\end{tabular}

Table 7

ONE-WAY ANOVA SUMMARY FOR THE PCS GROUPS

\begin{tabular}{|c|c|c|c|}
\hline $\begin{array}{l}\text { DEPENDENT } \\
\text { VARIABLE }\end{array}$ & df & $\bar{F}$ & $\bar{p}$ \\
\hline \multicolumn{4}{|l|}{ Age (yaers) } \\
\hline Between & 2 & $8.53^{-}$ & $=0.01$ \\
\hline Within & 119 & & \\
\hline \multicolumn{4}{|c|}{ HDL_CT (mg/dl) } \\
\hline Between & $2^{--}$ & 3.38 & $=0.05$ \\
\hline Within & 119 & & \\
\hline \multicolumn{4}{|l|}{ TI } \\
\hline Between & $2^{--}$ & $4.59^{-1}$ & $<0.05$ \\
\hline Within & 119 & & \\
\hline \multicolumn{4}{|l|}{$\overline{\mathrm{CQ}}$} \\
\hline Between & 2 & $35.84^{-}$ & $<0.01$ \\
\hline Within & 119 & & \\
\hline \multicolumn{4}{|l|}{$\overline{\mathrm{TC}}$} \\
\hline Between & 2 & 3.23 & $=0.05$ \\
\hline Within & 119 & & \\
\hline \multicolumn{4}{|l|}{$\overline{\mathrm{CH}}$} \\
\hline Between & 2 & 19.08 & $=0.01$ \\
\hline Within & 119 & & \\
\hline \multicolumn{4}{|l|}{$\overline{C Y}$} \\
\hline Between & 2 & 8.65 & $=0.01$ \\
\hline Within & $119^{\circ}$ & & \\
\hline \multicolumn{4}{|l|}{$\overline{E R}$} \\
\hline Between & $2^{--}$ & $19.31^{-}$ & $<0.01^{-}$ \\
\hline Within & 119 & & \\
\hline
\end{tabular}

significant for: $\mathrm{Tl}(\mathrm{t}(119)=3.06 ; \mathrm{p}=0.00), \mathrm{CQ}(\mathrm{t}(119)=$ 2.46; $p=0.00), C Y(t(119)=1.65 ; p=0.00)$.

Correlations of socio-demographics, hematological, biochemical, BIS and R-IPQ parameters between the three MCS groups (low-score, medium-score and high-score)

The independent between-groups ANOVA yielded a statistically significant effect for: age $(F(2.119)=4.80$; $p<0.05)$; PTH $(F(2.119)=3.26 ; p<0.05) ; C T(F(2.119)=$ 4.12; $p<0.05) ; \mathrm{TG}(\mathrm{F}(2.119)=3.29 ; \mathrm{p}<0.05) ; \mathrm{OH}(\mathrm{F}(2.119)$ $=4.32 ; p<0.05) ; \mathrm{OH} / \mathrm{ECW}(\mathrm{F}(2.119)=3.41 ; p<0.05) ; \mathrm{FTI}$ $(F(2.119)=3.89 ; p<0.05) ; \operatorname{TAS}(F(2.119)=5.96 ; p<0.05) ;$ $\operatorname{TAD}(F(2.119)=3.19 ; p<0.05) ; C Q(F(2.119)=38.41 ;$ $p<0.01) ; \operatorname{TC}(F(2.119)=6.08 ; p<0.05) ; \mathrm{CH}(F(2.119)=$ $22.10 ; p<0.01) ; C Y(F(2.119)=7.23 ; p<0.01) ; E R$ $(F(2.119)=32.15 ; p<0.01)$ (table 8).

The post-hoc test showed the following: the differences between the low-score MCS group and the high-score MCS group were statistically significant for: age $(\mathrm{t}(119)=10.69$; $\mathrm{p}=0.00)$, PTH $(\mathrm{t}(119)=-330.22 ; \mathrm{p}=0.01), \mathrm{CT}(\mathrm{t}(119)=$ 22.83; $\mathrm{p}=0.04), \mathrm{FTI}(\mathrm{t}(119)=3.66 ; \mathrm{p}=0.03), \mathrm{TAS}(\mathrm{t}(119)$ $=14.69 ; p=0.03), C Q(t(119)=4.63 ; p=0.00), T C(t(119)$ $=-1.63 ; \mathrm{p}=0.03), \mathrm{CH}(\mathrm{t}(119)=-4.81 ; \mathrm{p}=0.00), \mathrm{CY}(\mathrm{t}(119)$ $=1.75 ; p=0.00), \operatorname{ER}(t(119)=7.10 ; p=0.00)$. The differences between the low-score MCS group and the 
Table 8

ONE-WAY ANOVA SUMMARY FOR THE MCSGROUPS

\begin{tabular}{|c|c|c|c|}
\hline $\begin{array}{l}\text { DEPENDENT } \\
\text { VARIABLE }\end{array}$ & df & $\bar{F}$ & $\mathbf{p}$ \\
\hline \multicolumn{4}{|l|}{ Age (years) } \\
\hline Between & $2^{--}$ & $4.80^{\circ}$ & $=0.05$ \\
\hline Within & 119 & & \\
\hline \multicolumn{4}{|l|}{$\mathrm{PTH}_{\mathrm{i}}(\mathbf{n g} / \mathbf{l})$} \\
\hline Between & 2 & 3.26 & $<0.05$ \\
\hline Within & 119 & & \\
\hline \multicolumn{4}{|l|}{ CT (mg/dl) } \\
\hline Between & 2 & 4.12 & $=0.05$ \\
\hline Within & $119^{\circ}$ & & \\
\hline \multicolumn{4}{|l|}{ TG (mg/dl) } \\
\hline Between & 2 & 3.29 & $<0.05$ \\
\hline Within & 119 & & \\
\hline \multicolumn{4}{|l|}{$\mathrm{OH}(\mathrm{l})$} \\
\hline Between & $2^{-}$ & 4.32 & $<0.05$ \\
\hline Within & $119^{-}$ & & \\
\hline \multicolumn{4}{|l|}{ OH/ECW (\%) } \\
\hline Between & 2 & 3.41 & $<0.05$ \\
\hline Within & 119 & & \\
\hline \multicolumn{4}{|l|}{ FTI $\left(\mathrm{kg} / \mathrm{m}^{2}\right)$} \\
\hline Between & 2 & 3.89 & $<0.05$ \\
\hline Within & $119^{\circ}$ & & \\
\hline \multicolumn{4}{|l|}{ TAS (mmHg) } \\
\hline Between & 2 & 5.96 & $<0.05$ \\
\hline Within & $119^{\circ}$ & & \\
\hline \multicolumn{4}{|l|}{ TAD (mmHg) } \\
\hline Between & 2 & 3.19 & $=0.05$ \\
\hline Within & $119^{-}$ & & \\
\hline \multicolumn{4}{|l|}{ CQ } \\
\hline Between & 2 & 38.41 & $<0.01$ \\
\hline Within & $119^{-}$ & & \\
\hline \multicolumn{4}{|l|}{ TC } \\
\hline Between & 2 & 6.08 & $<0.05$ \\
\hline Within & $119^{-}$ & & \\
\hline \multicolumn{4}{|l|}{$\mathrm{CH}$} \\
\hline Between & 2 & 22.10 & $<0.01$ \\
\hline Within & 119 & & \\
\hline \multicolumn{4}{|l|}{$\mathrm{CY}$} \\
\hline Between & 2 & 7.23 & $=0.05$ \\
\hline Within & 119 & & \\
\hline \multicolumn{4}{|l|}{ ER } \\
\hline Between & 2 & 32.15 & $<0.01^{\circ}$ \\
\hline Within & 119 & & \\
\hline
\end{tabular}

medium-score MCS group were statistically significant for: $\mathrm{CT}(\mathrm{t}(119)=22.95 ; \mathrm{p}=0.00), \mathrm{TG}(\mathrm{t}(119)=52.16 ; \mathrm{p}=$ $0.01), \mathrm{OH}(\mathrm{t}(119)=-0.84 ; \mathrm{p}=0.00), \mathrm{OH} / \mathrm{ECW}(\mathrm{t}(119)=-$ $4.20 ; p=0.01), F T I(t(119)=3.24 ; p=0.01), C Q(t(119)$ $=2.30 ; p=0.00), \operatorname{TC}(t(119)=-0.99 ; p=0.01), C Y(t(119)$ $=0.73 ; p=0.04), \operatorname{ER}(t(119)=4.44 ; p=0.00)$. Finally, the differences between the medium-score MCS group and the high-score MCS group were statistically significant for: PTH $(\mathrm{t}(119)=-290.29 ; \mathrm{p}=0.03)$, TAS $(\mathrm{t}(119)=15.85 \mathrm{p}=$ $0.01), \operatorname{TAD}(\mathrm{t}(119)=7.59 ; \mathrm{p}=0.01), \mathrm{CQ}(\mathrm{t}(119)=2.33 ; \mathrm{p}$ $=0.00), \mathrm{CH}(\mathrm{t}(119)=-2.45 ; \mathrm{p}=0.01), \mathrm{CY}(\mathrm{t}(119)=1.02$; $p=0.02), E R(t(119)=2.65 ; p=0.00)$.

There are several reports that associate undernutrition in HD population with several nutritional and inflammatory markers, as decreased concentration of serum albumin, decreased concentration of serum cholesterol, low levels of BMI and elevated serum concentration of CRP $[10,16]$. Furthermore, malnutrition is an independentrisk factor for morbidity and mortality in ESRD patients [8,9]. Impaired QoL in these patients has a multifactorial origin, the baseline renal disease, the renal replacement therapy itself, the increased number of comorbidities, the systemic chronic inflammation and fluid overload status and the PEW syndrome being all accountable for a significantly decreased level of social, familial and professional interaction for ESRD patients receiving HD treatment $[9,10,12,17]$. In the last years, nutritional parameters evaluated through bioimpedance spectroscopy analysis, have gained a well-deserved place among the principal, fastest, easiest and reproducible techniques of accurate nutrition assessment $[14,18-26]$. Low muscle mass, expressed either as LTM $(\mathrm{kg})$ or LTI $\left(\mathrm{kg} / \mathrm{m}^{2}\right)$, a BIS-derived parameter, was found to be associated with higher mortality in HD patients $[12,13,17]$. In 2010, Huang et al. conducted a study on 1709 patients from the Hemodialysis Study, in order to evaluate the relationship between body composition and all-cause mortality and found that low muscle mass and low fat mass are predictors for all-cause mortality [12]. A recent 2018 study, performed on 48 HD patients showed that lower LTI $(p=0.013)$ and higher hsCRP levels ( $p=0.011$ ) were associated with higher mortality in the study's cohort [13].

Our study, performed in 122 stable HD patients, showed result consistent with previous studies findings. In the presentstudy, there was a statistically significant correlation between decreased illness perception and QoL scores and malnutrition and BIS-derived parameters. Therefore, the present analysis showed that the 122 included patients perceived an impaired illness perception and quality of life. The mean PCS score was negatively associated with a low serum albumin concentration, low serum total cholesterol and triglycerides concentration, low BMI, FTI and ATM/W levels, suggesting that undernutrition, expressed as low levels of nutritional markers, are associated to decreased quality of life in these patients, in terms of SF-36 physical domains evaluation. Also, mean MCS score was negatively associated with low serum total cholesterol and triglycerides concentration and with low FTI levels, suggesting a correlation between undernutrition and impaired quality of life, in terms of SF-36 mental assessment. In the same manner, the present study showed an association between nutritional markers and illness perception, and between R-IPQ scores and QoL scores, the two psychometric evaluation scales revealing consistent results.

The strengths of the present study consist in the multifactorial approach, analyzing socio-demographical, clinical, hematological, biochemical and BIS-derived parameters in relationship with two major psychometric evaluation scales. The limitation of the study are represented by the observational, cross-sectional design of the study that can not establish a causal relationship, and the relatively small sample size.

\section{Conclusions}

Well-conducted, large sample size cohort and randomized controlled trials are needed in order to establish a direct, strong, causality relationship between malnutrition-inflammation markers, BIS-derived parameters, QoL and mortality in HD patients, in order for efficient behavioral therapies to be part of a constant, longterm therapeutically approach in these patients.

\section{References}

1.HILL NR, FATOBA ST, OKE JL, et al., PLoS ONE, 2016, 11 (7), pp.118

2.PARFENI M, NISTOR I, COVIC A. et al., 2013, Nephrol Dial Transplant, $0, \mathrm{pp} .1-8$

3.DONCIU M, TASMOC A, DUMEA R, HOGAS S, VORONEANU L, SIRIOPOL MD, COVIC A, Rev. Med. Chir. Soc. Med. Nat., 2013, 117(4), pp.908-15 
4.LI YN, SHAPIRO B, KIM JC, ZHANG M, PORSZASZJ, BROSS R, FEROZE $U$ et al., Chronic Diseases and Translational Medicine, 2016, 2, pp.11019

5.SOLOMON SM, TANCULESCU O, SCUTARIU MM, PASARIN L, SUFARU IG, MARTU MA, LUCHIAN I, MARTU S. Int. J of Medical Dentistry, 2017, 21(4), pp.290-293

6.VEISA G, TASMOC A, NISTOR I, SEGAL L, SIRIOPOL D, SOLOMON SM, DONCIU MD, VORONEANU L, NASTASA A, COVIC A . Int Urology and Nephrology, 2017, 49(7), pp.1261-1266

7.SINCAR, C.D., IOANID, N., RUDNIC, I., MARTU, I., SOLOMON, S.M., PAVEL, L.L., REZUS, C., MARTU, S., CONDRATOVICI, C.P., Rev de Chim.(Bucharest), 68, no.3, 2017, p.605-607

8.VEISA, G., DONCIU, M.D., SEGAL, L., HURJUF, L., NISTOR, I., URSARESCU, I.G., MARTU, S., BURLEA, S.L., SOLOMON, S.M., Rev . Chim.(Bucharest), 67, no.1, 2016, pp. 103-5

9.HWANG SD, LEE JH, LEE SW. et al., 2018, Renal Failure, 40(1), pp.5159

10.BEDDHU S, CHEN X, WEI G, RAJ D, RAPHAEL KL, BOUCHER R. et al., 2017, Kidney International Reports, 2, pp.390-99

11.***http//www.bcm-fresenius.com/23.htm

12. HUANG CC, TIGHIOUART H, BEDDHU S, CHEUNG AK, DWYER JT et al., 2010, Kidney International, 77, pp.624-629

13.RYMARZ A, GIBRISKA J, ZAJBT M, PIECHOTA W, NIEMCZYK S, Ren Fail. 2018,40(1), pp.231-237
14.CASTELLANO S, PALOMARES I, MOISSL U, CHAMNEY P, CARRETERO D, CRESPO A et al., 2016, Nefrologia, 36(3), pp.268-274 15.WIZEMANN V, WABEL P, CHAMNEY P, et al., 2009, Nephrol Dial Transplat, 24 (5), pp. 1574-79

16.FOUQUE D, VENNEGOOR M, TER WEE P. et al., 2007, Nephrol Dial Transplant, 22(suppl 2), pp. ii45-ii87

17.ROSENBERG J, KISSOVA V, MAJERNIKOVA M. et al., 2014, J ournal of Renal Nutrition, 24(3), pp.172-176

18.WANG YW, LIN TY, PENG CH et al., 2017, Nutrients, 9(434), pp.1-9 19.J UMANCA,D.,GALUSCAN,A., PODARIU,A.C., et al, Rev.Chim. (Bucharest), 65, no.12, 2014, p.336-341

20.GRADINARU, I., IGNAT, L., DASCALU, C.G., SOROAGA, L.V., ANTOHE, M.E.,Rev.Chim.(Bucharest), 69, no.2, 2018, p.328-331

21.ASAFTEI, I.V, SANDU,I.G., MIHAIL,L,ET AL . Rev.Chim.(Bucharest), 66, no.3, 2015, p.336-341

22.ANTOHE, M.E., FORNA, AGOP, D., DASCALU, C.G., FORNA, N.C., Rev. Chim.(Bucharest), 69, no.2, 2018 p.521-524

23.MATEI, M.N., EARAR, K., TRINCA, L.C., Rev.Chim.(Bucharest), 67, no.4,2016, p.800-807

24.MATEI, M.N., CHISCOP, I, EARAR, K., et al., Rev.Chim.(Bucharest), 66, no.12,2015, p.2009-2012

25.MARECI, D., EARAR, K., ZETU, I, Mat.Plast., 52, no.2, 2015, pg, 150-153 26.TARANU, T., CONSTANTIN, M.M., TOADER, M.P,et al., Rev.Chim. (Bucharest), 69, no.9, 2018, p.2343-2345

$\overline{\text { Manuscript received } 21.09 .2018}$ 